\title{
Square Patterns and Quasi-patterns in Weakly Damped Faraday Waves
}

\author{
Wenbin Zhang ${ }^{1}$ and Jorge Viñals ${ }^{2}$ \\ ${ }^{1}$ Department of Chemical Engineering, Massachusetts Institute of Technology, Cambridge, \\ Massachusetts 02139 \\ ${ }^{2}$ Supercomputer Computations Research Institute, Florida State University, Tallahassee, Florida \\ 32306-4052, \\ and Department of Chemical Engineering, FAMU/FSU College of Engineering, Tallahassee, \\ Florida 31316
}

(October 31, 2018)

\begin{abstract}
Pattern formation in parametric surface waves is studied in the limit of weak viscous dissipation. A set of quasi-potential equations (QPEs) is introduced that admits a closed representation in terms of surface variables alone. A multiscale expansion of the QPEs reveals the importance of triad resonant interactions, and the saturating effect of the driving force leading to a gradient amplitude equation. Minimization of the associated Lyapunov function yields standing wave patterns of square symmetry for capillary waves, and hexagonal patterns and a sequence of quasi-patterns for mixed capillarygravity waves. Numerical integration of the QPEs reveals a quasi-pattern of eight-fold symmetry in the range of parameters predicted by the multiscale expansion.
\end{abstract}

PACS numbers: 47.35.+i, 47.54.+r, 47.20.Ky

Typeset using REVTEX 
When a fluid layer with a free upper surface is subjected to vertical oscillation, Faraday waves are observed [1,2]. In a large enough container and for low viscosity fluids, standing wave patterns of square symmetry are observed near threshold [3]. Based on amplitude equations that we derive below, the experimental observation of square patterns in the capillary-dominated regime is explained. We also predict that hexagonal and a sequence of quasi-patterns can be stabilized for the case of a sinusoidal driving force as a result of triad resonant interactions for mixed capillary-gravity waves [4]. To our knowledge, this is the first theoretical derivation that starting from a realistic model of the fluid shows that a quasi-crystalline pattern is a stable steady state, and corroborates the conjecture of Newell and Pomeau [5] on the existence of the so-called "turbulent crystals".

Pattern-forming instabilities occur in a variety of extended nonlinear systems. The emergence of spatial patterns close to onset of the instability can often be described by amplitude equations [2,6]. However, for near-Hamiltonian (or weakly dissipative) systems, there is no general agreement on how dissipation should be incorporated into the amplitude equation formulation. Previous work on Faraday waves was based on amplitude equations for a purely Hamiltonian system, to which linear and nonlinear damping terms were added by introducing a dissipation function [7,8]. In this approach, linear dissipative effects in the original system contribute only to linear damping terms in the associated amplitude equations, while nonlinear damping terms result entirely from nonlinear dissipative effects. Such an approach has contributed to the general belief that for near-Hamiltonian systems, nonlinear saturation of the linear instability does not occur if only linear dissipative effects are considered, and weak nonlinear dissipative or other higher order effects are needed for nonlinear saturation [2, 9]. In this paper, we show that in the case of weakly damped parametric surface waves linear dissipative effects do contribute to the nonlinear damping terms in the amplitude equation, and that they alone can saturate the parametric instability. In addition, the experimental observation of square patterns in capillary-dominated regime is naturally explained without having to invoke poorly understood nonlinear dissipative effects, or higher order terms in the amplitude equation. 
The basic difference with previous studies [0, 8 is that although the bulk flow does remain potential, it is modified by a rotational viscous boundary layer near the free surface that has to be explicitly incorporated into the analysis 10 12. When the thickness of the viscous boundary layer is small compared to the typical wavelength of the pattern, the weak effects due to viscosity can be taken into account by introducing effective boundary conditions for the otherwise potential bulk flow. This is the basic idea of the quasi-potential approximation introduced below. We first expand the equations governing the motion of an incompressible viscous fluid and the appropriate boundary conditions at the free surface in the small thickness of the free surface boundary layer, $\delta$. The resulting equations are further simplified by recasting them in a nonlocal form that involves the flow variables on the free surface only; thus eliminating the need to explicitly solve for the flow in the bulk [12]. Let $z$ be the normal direction to the surface at rest and $g(t)=-g_{0}-g_{z 0} \sin \Omega t$ the driving force where $g_{0}$ is the constant acceleration of gravity, and $\Omega$ and $g_{z 0}$ are the angular frequency and the amplitude of the driving force respectively. We choose $1 / \omega_{0} \equiv 2 / \Omega$ as the unit of time and $1 / k_{0}$ as the unit of length, with $k_{0}$ defined by $\omega_{0}^{2}=g_{0} k_{0}+\Gamma k_{0}^{3} / \rho$, the linear dispersion relation for surface waves, where $\Gamma$ is the surface tension and $\rho$ the density of the fluid. Then the dimensionless, nonlocal and quasi-potential equations read [12],

$$
\begin{array}{r}
\partial_{t} h(\mathbf{x}, t)=\gamma \nabla^{2} h+\hat{\mathcal{D}} \Phi-\nabla \cdot(h \nabla \Phi)+\frac{1}{2} \nabla^{2}\left(h^{2} \hat{\mathcal{D}} \Phi\right) \\
-\hat{\mathcal{D}}(h \hat{\mathcal{D}} \Phi)+\hat{\mathcal{D}}\left[h \hat{\mathcal{D}}(h \hat{\mathcal{D}} \Phi)+\frac{1}{2} h^{2} \nabla^{2} \Phi\right] \\
\partial_{t} \Phi(\mathbf{x}, t)=\gamma \nabla^{2} \Phi-\left(G_{0}-\Gamma_{0} \nabla^{2}\right) h-4 f h \sin 2 t \\
+\frac{1}{2}(\hat{\mathcal{D}} \Phi)^{2}-(\hat{\mathcal{D}} \Phi)\left[h \nabla^{2} \Phi+\hat{\mathcal{D}}(h \hat{\mathcal{D}} \Phi)\right] \\
-\frac{1}{2}(\nabla \Phi)^{2}-\frac{\Gamma_{0}}{2} \nabla \cdot\left(\nabla h(\nabla h)^{2}\right),
\end{array}
$$

where $\mathbf{x}=(x, y), \nabla=\left(\partial_{x}, \partial_{y}\right), h$ is the surface displacement away from planarity, $\Phi$ is the value of velocity potential at the free surface (the surface velocity potential), $\gamma=2 \nu k_{0}^{2} / \omega_{0}$ is a linear damping coefficient $\left(\gamma \sim \delta^{2} \ll 1\right), G_{0}=g_{0} k_{0} / \omega_{0}^{2}, \Gamma_{0}=\Gamma k_{0}^{3} /\left(\rho \omega_{0}^{2}\right)$, and $f=$ $g_{z 0} k_{0} /\left(4 \omega_{0}^{2}\right)$ is the driving amplitude, and $\hat{\mathcal{D}}$ is a Fourier-integral operator, which is defined 
for an arbitrary function $u(\mathbf{x})$ by $\hat{\mathcal{D}} u(\mathbf{x})=\int_{-\infty}^{\infty}|\mathbf{k}| \hat{u}(\mathbf{k}) \exp (i \mathbf{k} \cdot \mathbf{x}) d \mathbf{k}$ with $\hat{u}(\mathbf{k})$ the Fourier transform of $u(\mathbf{x})$. Notice that $G_{0}+\Gamma_{0}=1$ by definition. Only those viscous terms that are linear in the surface variables are retained in Eqs. (11) and (2). Nonlinear viscous terms, which were referred to as nonlinear dissipative effects in the introduction, have been neglected. Both the asymptotic analysis that follows and the numerical results presented later are based on Eqs. (1) and (21).

Linear analysis indicates that for $\gamma \ll 1$ the planar surface becomes unstable at $f_{c}=\gamma$, and the critical wavenumber is $k_{0}=1$. We seek nonlinear standing wave solutions near threshold $(\varepsilon \equiv(f-\gamma) / \gamma \ll 1)$ and expand Eqs. (1) and (2) in $\varepsilon^{1 / 2}$ with multiple time scales, $h(\mathbf{x}, t, T)=\varepsilon^{1 / 2} h_{1}(\mathbf{x}, t, T)+\varepsilon h_{2}+\varepsilon^{3 / 2} h_{3}+\cdots$ and $\Phi(\mathbf{x}, t, T)=\varepsilon^{1 / 2} \Phi_{1}(\mathbf{x}, t, T)+\varepsilon \Phi_{2}+$ $\varepsilon^{3 / 2} \Phi_{3}+\cdots$, where $T=\varepsilon t$. At $\mathcal{O}\left(\varepsilon^{1 / 2}\right)$, we consider a set of $N$ standing wave modes with critical wavevectors $\pm \hat{k}_{j}$; then the linear solution reads,

$$
\begin{gathered}
h_{1}=\left(\cos t+\frac{f}{4} \sin 3 t\right) \sum_{j=1}^{N}\left[A_{j}(T) \exp \left(i \hat{k}_{j} \cdot \mathbf{x}\right)+c . c .\right], \\
\Phi_{1}=\left(-\sin t+f \cos t+\frac{3 f}{4} \cos 3 t\right) \\
\quad \times \sum_{j=1}^{N}\left[A_{j}(T) \exp \left(i \hat{k}_{j} \cdot \mathbf{x}\right)+\text { c.c. }\right],
\end{gathered}
$$

where the complex amplitudes $A_{j}$ are assumed to vary in the slow time scale $T$. Notice that the linear solution contains not only the subharmonic responses but also the terms that are proportional to the driving force $f$. These latter terms arise because $f$ is finite for the expansion in $\varepsilon$. Terms that are of higher order in $f$ have been neglected. In what follows, we shall replace $f$ in the linear solution by $\gamma$ since $f=\gamma(1+\varepsilon)$.

At $\mathcal{O}(\varepsilon)$, there is no solvability condition; however there are resonant interactions that have to be taken into account. A particular solution for $h_{2}$ can be written as

$$
\begin{aligned}
h_{2}= & \sum_{j, l=1}^{N}\left\{H_{j l}(t)\left[A_{j} A_{l} \exp \left(i\left(\hat{k}_{j}+\hat{k}_{l}\right) \cdot \mathbf{x}\right)+c . c .\right]\right. \\
& \left.+H_{j,-l}(t)\left[A_{j} A_{l}^{*} \exp \left(i\left(\hat{k}_{j}-\hat{k}_{l}\right) \cdot \mathbf{x}\right)+\text { c.c. }\right]\right\},
\end{aligned}
$$

where the $H_{j l}(t)$ are unknown functions that satisfy, 


$$
\begin{aligned}
& \partial_{t t} H_{j l}+2 \gamma \sqrt{2\left(1+c_{j l}\right)} \partial_{t} H_{j l}+\left[G_{0}+2 \Gamma_{0}\left(1+c_{j l}\right)\right] \\
& \times \sqrt{2\left(1+c_{j l}\right)} H_{j l}=F_{j l}^{(1)} \cos 2 t+F_{j l}^{(2)} \sin 2 t+\cdots,
\end{aligned}
$$

where $c_{i j} \equiv \cos \theta_{i j}=\hat{k}_{j} \cdot \hat{k}_{l}$, and $F_{j l}^{(1)}$ and $F_{j l}^{(2)}$ are proportional to the amplitudes $A_{j} A_{l}$. Equation (6) is the equation of an additively forced harmonic oscillator with friction. When the "natural" frequency of the "oscillator" equals to the driving frequency, resonance occurs. This condition reads, $\left[G_{0}+2 \Gamma_{0}\left(1+c_{j l}\right)\right] \sqrt{2\left(1+c_{j l}\right)}=4$. Due to the nonzero damping coefficient, this resonance results in a finite value for $H_{j l}$ that is inversely proportional to the damping coefficient. Since the RHS of Eq. (6) is proportional to $A_{j} A_{l}$, there are three waves involved in this resonance, namely, standing wave modes $A_{j}$ and $A_{l}$, and mode $B$ with wavevector $\hat{k}_{j}+\hat{k}_{l}$. Therefore, Eq. (6) describes a three-wave resonant interaction. Note that the wavenumber for mode $B$ is away from the critical wavenumber $k_{0}=1$; thus mode $B$ is a linearly stable mode. The relevance of triad resonant interactions to pattern selection can be understood intuitively. Since the resonant growth of linearly damped mode $B$ is at the expense of the reduction of the amplitudes $A_{j}$ and $A_{l}$, the growth of modes $A_{j}$ and $A_{l}$ are less favored than any other mode $A_{m}$ that does not participate in a triad resonant interaction. In other words, the system tries to avoid critical modes that participate in triad resonant interactions. As shown below, triad resonant interactions strongly influence pattern selection through coefficients of cubic nonlinear terms in the amplitude equations in a way that is consistent with the above argument. Finally, for $\Gamma_{0}<1 / 3$, triad resonance is not possible. For $\Gamma_{0}=1 / 3$, wavevectors of the three resonating waves are in the same direction $\left(\theta_{j l}^{(r)}=0\right)$. As $\Gamma_{0}$ is further increased, $\theta_{j l}^{(r)}$ also increases. For purely capillary waves $\left(\Gamma_{0}=1\right), \theta_{j l}^{(r)}$ reaches the maximum value of $\theta_{j l}^{(r)} \approx 74.9^{\circ}$ or $c_{j l}=2^{1 / 3}-1$.

At $\mathcal{O}\left(\varepsilon^{3 / 2}\right)$, we obtain the standing wave amplitude equations (SWAEs) from a non-trivial solvability condition,

$$
\frac{\partial A_{j}}{\partial T}=\gamma A_{j}-\left[\gamma g(1)\left|A_{j}\right|^{2}+\gamma \sum_{l=1(l \neq j)}^{N} g\left(c_{j l}\right)\left|A_{l}\right|^{2}\right] A_{j},
$$

where $j=1,2, \cdots, N$, and $g(1)$ and $g\left(c_{j l}\right)$ are given in Ref. [12]. There are two kinds of contributions to $g(1)$ and $g\left(c_{j l}\right)$. One is from the linear viscous terms in Eqs. (1) and (2). 
The other is due to the parametric driving force and proportional to the driving amplitude $f$. These two kinds of contributions appear together in Eq. (7) since we have set $f=\gamma$ at the linear order. The latter contribution is directly related to the terms proportional to $f$ in the linear solution (Eqs. (3) and (困)), and it provides an amplitude-limiting effect. The nonlinear interactions between the primary subharmonic modes and terms related to the driving force in the linear solution produce terms that are out of phase by $\pi / 2$ with the primary subharmonic mode, and thus can contribute to saturate the wave amplitude. An important point is that this amplitude-limiting effect results from the forcing term, but not from a dissipative term. As a result, this effect is also important even for Hamiltonian systems.

It can be shown that $g(1)>0$ [12], which indicates the bifurcation to the standing wave state is supercritical. We rescale the amplitudes as $\sqrt{g(1)} A_{j}$ and the coefficients as $g\left(c_{j l}\right) / g(1)$. In what follows, we shall only refer to the scaled amplitudes and the scaled coefficients; but use the same notation for them as for the unscaled ones. Note that the scaled coefficient $g\left(c_{j l} \rightarrow \pm 1\right)=2$. Figure [ for two different values of the damping coefficient $\gamma$ and $\Gamma_{0}=1$. The maxima in $g\left(c_{j l}\right)$ around $c_{j l}=0.26\left(\theta_{j l}=74.9^{\circ}\right)$ correspond to the triad resonance for purely capillary waves. The function $g\left(c_{j l}\right)$ for capillary-gravity waves of $\Gamma_{0}=1 / 3$ is shown in Fig. 1(c) and (d). Since the triad resonant interaction occurs among waves with their wavevectors in the same direction when $\Gamma_{0}=1 / 3$, the resonant peaks (or maxima) are at $c_{j l}= \pm 1$. Instead of large peaks at $c_{j l}= \pm 1$, we see that $g\left(c_{j l}\right)$ has a wide flat region around $c_{j l}=0$ and reaches very small positive values for small $\gamma$ due to scaling. In all cases, the effect of triad resonance is weaker for larger values of $\gamma$ as expected. 


\section{FIGURES}
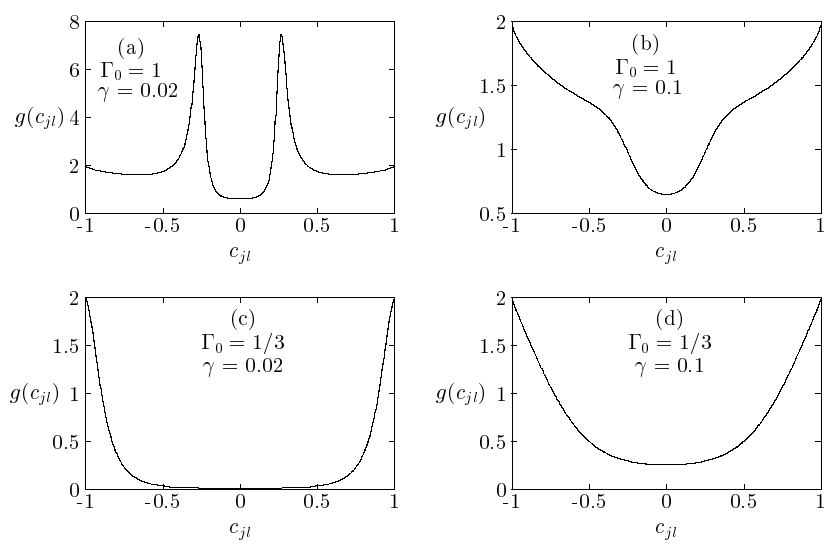

FIG. 1. The coefficient $g\left(c_{j l}\right)$ as a function of $c_{j l}$ for purely capillary waves $\left(\Gamma_{0}=1\right)$ with the linear damping coefficient $\gamma=0.02(\mathrm{a})$, and $\gamma=0.1(\mathrm{~b})$. The same coefficient for gravity-capillary waves of $\Gamma_{0}=1 / 3$ with $\gamma=0.02(\mathrm{c})$ and $\gamma=0.1(\mathrm{~d})$.

The issue of pattern selection can be discussed by noting that Eq. (17) is of gradient form $1 / \gamma \partial_{T} A_{j}=-\partial \mathcal{F} / \partial A_{j}^{*}$. Apart from the trivial solution of $A_{j}=0$ for $j=1, \cdots, N$, Eq. (7) has a family of stationary solutions differing in the total number of standing waves $N$ for which $A_{j} \neq 0$. By considering the case in which the magnitudes of all standing waves are the same, Eq. (7) has the following solutions, $\left|A_{j}\right|=|A|=\left(1+\sum_{l=1(l \neq j)}^{N} g\left(c_{j l}\right)\right)^{-1 / 2}$. The Lyapunov function for these solutions are, $\mathcal{F}=-\frac{N}{2}|A|^{2}=-\frac{N}{2} /\left[1+\sum_{l=1(l \neq j)}^{N} g\left(c_{j l}\right)\right]$. For $N=1$ (parallel roll solution), $\mathcal{F}_{1}=-\frac{1}{2}$. For $N=2$, we have either square $\left(c_{12}=0\right)$ or rhombic $\left(c_{12} \neq 0\right)$ patterns with $\mathcal{F}_{2}=-1 /\left(1+g\left(c_{12}\right)\right)$. If we consider only regular patterns, i.e. pattern structures for which the angle between any two adjacent wavevectors $\mathbf{k}_{j}$ and $\mathbf{k}_{j+1}$ is the same and amounts to $\pi / N$, we have either hexagonal or triangular patterns for $N=3$. Regular patterns for $N \geq 4$ are two-dimensional quasi-crystalline patterns (or quasi-patterns [13]). A quasi-pattern has long-range orientational order but no spatial periodicity, thus analogous to a quasi-crystal in solid state physics. Such patterns have been already observed in experiments of Faraday waves in systems driven by two carefully chosen frequency components [13], but not in the single frequency case analyzed here. Fig. 2 shows the Lyapunov function $\mathcal{F}_{N}$ as a function of $\gamma$ for $N=1,2,3,4,5,6,7,8$, and $\Gamma_{0}=1 / 3$ and 1 . 
For $\Gamma_{0}=1$, patterns of square symmetry $(N=2)$ have the lowest values of $\mathcal{F}_{N}$ for all values of $\gamma<0.2$, in agreement with experiments. However, patterns of different symmetries are favored in different ranges of $\gamma$ for $\Gamma_{0}=1 / 3$. This can be understood qualitatively by noting that the self-interactions of the critical modes are less favored than interactions among them. Consequently, pattern structures with large $N$ are favored.
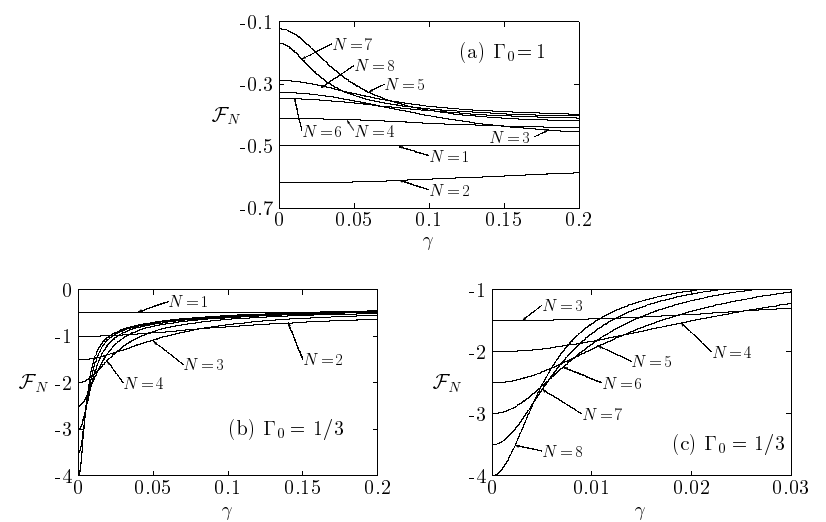

FIG. 2. The values of the Lyapunov function $\mathcal{F}_{N}(N=1,2,3,4,5,6,7,8)$ as a function of $\gamma$ for purely capillary waves $\left(\Gamma_{0}=1\right)$ in $(\mathrm{a})$, and for capillary-gravity waves of $\Gamma_{0}=1 / 3$ in (b). Part of (b) with small $\gamma$ is shown in (c).

We next present results from numerical solutions of the QPEs (Eqs. (11) and (2)) to check the stability of the quasi-patterns described above. An analytic stability calculation is far too involved. We use a Fourier-Galerkin spectral method [14] with periodic boundary conditions in a square domain. Time discretization is of second order. We use the trapezoidal scheme for the linear terms and the second order Adams-Bashforth scheme for nonlinear terms. The nonlinear terms are calculated by a pseudospectral method by using Fast Fourier Transforms. Most of the numerical studies are done for a system size of $64 \pi \times 64 \pi$, or $32 \lambda_{0} \times 32 \lambda_{0}$ with the critical wavelength $\lambda_{0}=2 \pi / k_{0}=2 \pi$, and a total number of 256 Fourier modes is used for each axis. We use a time step $\Delta t=0.04-0.1$. Extensive numerical studies have been performed for different values of the three dimensionless parameters $\Gamma_{0}, \gamma$, and $\varepsilon$ in the QPEs.

FIG. 3. Configuration of $h(\mathbf{x}, t)$ at $t=32000$ is shown in gray scale for $\gamma=0.02, \Gamma_{0}=1 / 3$, and $\varepsilon=0.1$. 
In the case of purely capillary waves $\left(\Gamma_{0}=1\right)$, Eqs. (11) and (2) are integrated for $\gamma=0.02,0.05,0.1,0.2$, and small values of $\varepsilon=0.02,0.05,0.1$, from a random initial condition (gaussianly distributed with zero mean and a variance of $\left.10^{-4}\right)$ for field $h(\mathbf{x}, t=0)$, and zero values for $\Phi(\mathbf{x}, t=0)$. Asymptotically regular patterns of standing waves with square symmetry are obtained for all the above parameters; thus verifying the results of the asymptotic analysis presented earlier, and again in agreement with experimental observation. For capillary-gravity waves at $\Gamma_{0}=1 / 3$, the QPEs are integrated for three different values of $\gamma=0.02,0.05$, and 0.1 at $\varepsilon=0.05$, with the same initial conditions. For $\gamma=0.1$, the long time configuration of $h(\mathbf{x}, t)$ is of approximate square symmetry. For $\gamma=0.05$, the long time configuration of $h(\mathbf{x}, t)$ is of approximate hexagonal symmetry. For $\gamma=0.02$, and to avoid finite size effects (expected to be stronger for smaller damping coefficient $\gamma$ [12,13]), we have performed numerical studies for a system of size $64 \lambda_{0} \times 64 \lambda_{0}$ with 512 Fourier modes for each axis and a time step $\Delta t=0.1$. The initial condition for $h$ is a set of gaussianly distributed random numbers with zero mean and a variance of $10^{-6}$, and $\Phi$ is set to zero initially. The configuration of $h(\mathbf{x}, t)$ at $t=32000$ is shown in Fig. 3. The structure factor of this configuration has been computed and has eight peaks, which correspond to eightfold symmetry of the standing wave pattern. This can also be seen by viewing Fig. 3 at a glancing angle. Thus, the basic prediction of the SWAEs is confirmed. Finally, we remark that the possible confirmation of stable pattern structures of even lower symmetry that are predicted by the SWAEs for smaller values of the damping coefficient $\gamma$ would require systems of larger size.

This work was supported by the Microgravity Science and Application Division of the NASA under Contract No. NAG3-1284. This work was also supported in part by the Supercomputer Computations Research Institute, which is partially funded by the U.S. Department of Energy, contract No. DE-FC05-85ER25000. 


\section{REFERENCES}

[1] J. Miles and D. Henderson, Annu. Rev. Fluid Mech. 22, 143 (1990).

[2] M. Cross and P. Hohenberg, Rev. Mod. Phys. 65, 851 (1993); A. Newell, T. Passot, and J. Lega, Annu. Rev. Fluid Mech. 25, 399 (1993).

[3] R. Lang, J. Acoust. Soc. Amer. 34, 6 (1962); A. Ezerskii, M. Rabinovich, and I. Starobinets, Zh. Eksp. Teor. Fiz. 91, 2070 (1986), [Sov. Phys. JETP 64, 1228 (1986)]; N.B. Tufillaro, R. Ramshankar, and J. Gollub, Phys. Rev. Lett. 62, 422 (1989).

[4] Hexagonal patterns have been recently observed experimentally by W.S. Edwards and J.P. Gollub (private communication).

[5] A. Newell and Y. Pomeau, J. Phys. A 26, L429 (1993).

[6] A. Newell and J. Whitehead, J. Fluid Mech. 38, 279 (1969); L.A. Segel, J. Fluid Mech. 38, 203 (1969); M.C. Cross, Phys. Fluids 23, 1727 (1980).

[7] S. Milner, J. Fluid Mech. 225, 81 (1991).

[8] J. Miles, J. Fluid Mech. 248, 671 (1993).

[9] P. Coullet, T. Frisch, and G. Sonnino, Phys. Rev. E 49, 2087 (1994).

[10] T. Lundgren and N. Mansour, J. Fluid Mech. 194, 479 (1988).

[11] K. Ruvinsky, F. Feldstein, and G. Freidman, J. Fluid Mech. 230, 339 (1991); M.S. Longuet-Higgins, J. Fluid Mech. 240, 659 (1992).

[12] W. Zhang, Ph.D. thesis, Florida State University, 1994.

[13] W. Edwards and S. Fauve, J. Fluid Mech. 278, 123 (1994).

[14] C. Canuto, M. Hussaini, A. Quarteroni, and T. Zang, Spectral Methods in Fluid Dynamics (Springer Verlag, New York, 1986). 
This figure "wz3.fig3.gif" is available in "gif" format from: http://arxiv.org/ps/patt-sol/9603005v1 